\title{
Público visitante de Museos. El caso en Antioquia- Colombia
}

\section{Public visiting museums. A case in Antioquia Colombia}

DOI: $10.46932 / \mathrm{sfjdv2n4-060}$

Received in: May 1st, 2021

Accepted in: Jun 30th, 2021

\author{
Mónica María Valle Flórez \\ Doctora en Estudios Científicos y Sociales - ITESO \\ Politécnico Colombiano Jaime Isaza Cadavid, \\ Carrera 48 N.7-151 - Medellín Colombia \\ E-mail: mmvalle@elpoli.edu.co \\ Claudia María Giraldo Velásquez \\ Doctora en Turismo, Universidad de San Martín de Porres, Lima-Perú \\ Institución Universitaria Colegio Mayor de Antioquia, \\ E-mail: cgiraldo@colmayor.edu.co \\ Sandra Zapata Aguirre \\ Doctora en Gestión de Infraestructuras Aeronáuticas, Universidad de Enna, Italia. \\ Institución Universitaria Colegio Mayor de Antioquia, \\ E-mail: sandra.zapata@colmayor.edu.co
}

\section{RESUMEN}

Los museos son unos de los espacios culturales más mediáticos del panorama social contemporáneo, de allí su interés por comprender las necesidades, valores y expectativas de sus visitantes para pensar estrategia de marketing, comunicación e innovación.

En este artículo se presenta el análisis del público visitante de un Museo en el Departamento de AntioquiaColombia. El estudio se realizó en alianza estratégica entre el Grupo de investigación en ComunicaciónGIC, del Politécnico Colombiano Jaime Isaza Cadavid y el Grupo Empresarial y Turístico GIET, de la Institución Universitaria Colegio Mayor de Antioquia. Se utilizó metodología mixta, se aplicaron cuestionarios y se realizaron grupos focales. En esta ponencia se presenta solo los resultados cuantitativos. En el estudio se encuentra que la ocupación de la mayoría de los visitantes es estudiante, seguida de empleado y trabajadores independientes. Emergen como nuevo perfil de visitante la empleada doméstica y el jubilado. El visitante nacional asiste con su familia o con amigos. Prefiere espacios en los que pueda conversar a los que se requiera silencio. Los extranjeros tienen alto nivel educativo, prefieren una experiencia museística cognitiva, introspectiva o artística, el nacional opta por la experiencia social. Se presenta una tendencia por experiencias de ocio que permitan la relajación y el entretenimiento. A los museos se les presentan oportunidades y retos que les implica cambios sustanciales de su concepción clásica de museo.

Palabras clave: Museos, públicos, Antioquia, cultura, entretenimiento, ocio.

\begin{abstract}
Museums are one of the most media cultural spaces of contemporary social landscape, hence the interest in generating strategies to connect, loyalty and attracting public. In this paper the analysis of the visitors of a museum in the Department of Antioquia, Colombia is presented. The study was conducted in strategic partnership between the Research Group Communication- GIC, the Politecnico Colombiano Jaime Isaza
\end{abstract}


Cadavid and GIET and Tourism Business Group, of the University Institution Greater School of Antioquia. mixed methodology was used, were applied questionnaires and focus groups were conducted. This paper presents quantitative results alone.

The study found that the occupation of most visitors is a student, followed by employee and selfemployed. Emerge as a new visitor profile and the maid retired. The national visitor attends with family or friends. He prefers spaces where you can talk to those requiring silence. Foreigners attending alone, have high educational level. Unlike foreigners who prefer a more cognitive, introspective or artistic museum experience, the national opt for the social experience. a tendency for leisure experiences that allow relaxation and entertainment is presented. A museums are present both opportunities and challenges involved were substantial changes in its classical conception of the museum.

Keywords Museums, public, Antioquia, culture, entertainment, leisure.

\section{INTRODUCCIÓN}

La misión de los museos está cambiando de manera significativa, tradicionalmente se han dirigido, a la adquisición, conservación, investigación y exposición de objetos, ahora se han convertido en entidades de conocimiento y ocio (Capriotti,2013). Para Hood (1983) es preciso mostrar el museo como un lugar donde se puede interactuar en familia, disfrutar y relajarse con los amigos, no solamente como un lugar donde se aprende o se encuentran retos.

Los museos en Antioquia tienen un papel cada vez más significativo como centro dinamizador de la cultura y como entidades de servicio para el ciudadano local y para el turista. La capacidad de los museos para identificar y desarrollar sus potencialidades es un buen fundamento para la exitosa consecución de sus objetivos. En Colombia, la política nacional y de turismo cultural reconocen como elemento esencial en la gestión de estos espacios, el conocimiento preciso de su demanda a través de investigaciones que permitan orientar sus estrategias de gestión territorial y acciones promocionales (ECD, 2010). Se sabe que los colombianos asisten poco a los museos, por debajo de la media nacional se encuentra la región central, de la cual hace parte Antioquia. Esta región, el estudio de públicos y comunicación en museos, se lidera desde la Facultad de Arte de la Universidad de Antioquia, lo que sugiere abordajes interdisciplinarios para el análisis de los museos, en tanto dichos estudios se generaban principalmente desde las Facultades de comunicación.

En este panorama es necesario que los museos "desarrollen la audiencia", lo que en palabras de Walts (2006) es conocer mejor las necesidades de los visitantes. Margaret Hart Robertson, coordinadora de la ULPGC del Proyecto de la Universidad Metropolitana de Londres, sugiere que es necesario que el

\footnotetext{
${ }^{1}$ Esto es perfeccionar y mejorar la comunicación con los visitantes, lograr una audiencia que sea frecuente, convertir los no visitantes en visitantes, a los visitantes en visitantes frecuentes y a los asistentes regulares del museo en seguidores, mejorar el acceso, ofrecer múltiples experiencias, involucrar a los visitantes (manos y mentes), establecer una red activa con especiales grupos de destinatarios. Idem.
} 
museo se transforme en espacio 'vivo' para nuevas formas de ver, en lugar de encuentro para nuevas comunidades, en vez de espacio 'muerto', protector del pasado.

El estudio que se presenta tuvo entre sus objetivos determinar el perfil de los visitantes de un museo de la región antioqueña de Colombia; su lugar de procedencia, conocer los medios por los que se entera de las acciones del museo, el uso del tiempo libre, entre otros aspectos.

\section{MARCO TEÓRICO}

En el contexto de la sociedad del mercado los museos deben posicionarse como cualquier empresa que presta un servicio. Por ello requiere contar con un área que realice investigación de sus públicos, estudie las tendencias, evalúe las exposiciones, mida la satisfacción, diseñe el lanzamiento publicitario y de comunicación, analice la fidelización de la visita, la captación de nuevos socios y amigos del museo (Rodríguez, 2002). El estudio del potencial de las audiencias de los museos comprende tres aspectos, a saber: los tipos de públicos, los programas públicos y educativos y el plan de comunicación (Asensio \& Pol; 2007).

Para Hooper-Greenhill (2000) la comunicación institucional es importante en la gestión de los públicos de los museos. Entre las principales técnica de comunicación en estos están la publicidad, el marketing de comunicaciones, la identidad visual y la comunicación electrónica. Los museos pueden crear un marketing adecuado de mensajes de comunicación, influenciando el proceso de toma de decisiones, siempre y cuando conozcan las motivaciones de los visitantes, comprendiendo las necesidades básicas, valores y expectativas de estos (Slater,2006).

Los profesionales de la comunicación y el marketing en los museos necesitan ser conscientes de que los visitantes cambian constantemente sus percepciones. Esto les permitirá desarrollar múltiples estrategias de interpretación para dar cabida a todos estos grupos, posicionar sus productos, desarrollar la comunicación pertinente de los mensajes para su comercialización y elevar los niveles de satisfacción así como comprender las respuestas emocionales de estos (Pincus, 2004, citado por Slater, A. (2006 ). La más de las veces el marketing y la comunicación en los museos se orienta exclusivamente al contenido, bajo la presunción de que este es lo más importante. Esta es una concepción tradicional, ya que la comunicación estratégica debe dar prioridad también a otros asuntos como las relaciones con los públicos, en tal sentido debe pesarse el museo como entidad de conocimiento y de ocio, y por tanto las relaciones con sus públicos debe ser estrecha y de igual importancia que las colecciones (Capriotti, 2013). 


\section{METODOLOGÍA}

Para el estudio se diseñó un cuestionario, se realizó la prueba piloto de este y se aplicó a 400 visitantes, 200 nacionales y 200 extranjeros $^{2}$. El cuestionario fue estructurado para obtener información con respecto a: aspectos sociodemográficos también del uso de medios electrónicos, aspectos de la visita, aspectos técnicos y logísticos del Museo, nivel de satisfacción entre otros. Se realizaron preguntas abiertas y cerradas.

\section{RESULTADOS}

Después de la sistematización de datos y su interpretación se determinan los siguientes perfiles de público visitante del museo:

\subsection{PÚBLICO LOCAL}

Perfiles 1: Estudiante. Mujeres y hombres, entre 18 y 34 años, con nivel educativo de secundaria y universitario $^{3}$. Los de secundaria se ubican en los estratos 1,2 y 3. A diferencia de los universitarios que pertenecen a estratos 4, 5 y 6 . Hace también parte de este perfil los niños y niñas de primaria de estratos 1, 2 y 3. Perfil 2. Mujeres y Hombres entre 35 y los 54 años, técnicos y tecnólogos, de estratos 1, 2 y 3.

La presencia del estrato 1, 2 y 3 a nivel de estudiantes y personal en general quizás se deba a los apoyos estatales para visita de públicos a museos, estos consisten en que pueden ingresar de manera gratuita, con solo presentar la factura de servicios en la que se indica el estrato socio económico. Es de aclarar que el museo para el que se efectúo el estudio es de carácter privado y tienen un costo por el ingreso al recinto.

- Público local emergente: En cuanto a ocupaciones emergentes en términos de visitantes están el de las empleadas domésticas y la de los jubilados.

- Público local potencial. Estudiantes, ejecutivos y profesionales de estratos 4, 5 y 6. Artistas, profesores y personal del sector de la cultura. Se encuentra que el $66 \%$ de quienes visitan el Museo son solteros.

\section{- Procedencia del Visitante de Medellín}

\footnotetext{
${ }^{2}$ Meses de julio- agosto de 2014.

${ }^{3}$ Este dato resulta positivo en tanto en el 2013 se encontraba que un alto porcentaje de Universitarios de Medellín, no visitaban el Museo) Informe "Análisis de Público Universitario para el Museo de Antioquia” (2013). Dra. Mónica Valle FlórezPolitécnico Colombiano Jaime Isaza Cadavid. Dra. Claudia Giraldo Velásquez . Dra. Sandra Zapata Aguirre. Institución Universitaria Colegio Mayor de Antioquia
} 
En relación a los visitantes del Museo que proceden de Medellín se encuentra que hay un alto porcentaje $49 \%$ que no reportan este dato, y un 25\% que indica otros. El 5\% de los visitantes son del barrio Manrique, el 4\% de Robledo, 3\% de Aranjuez, el 2\% de Belén. La presencia de personas de estratos 1,2 y 3 en el Museo se hace evidente.

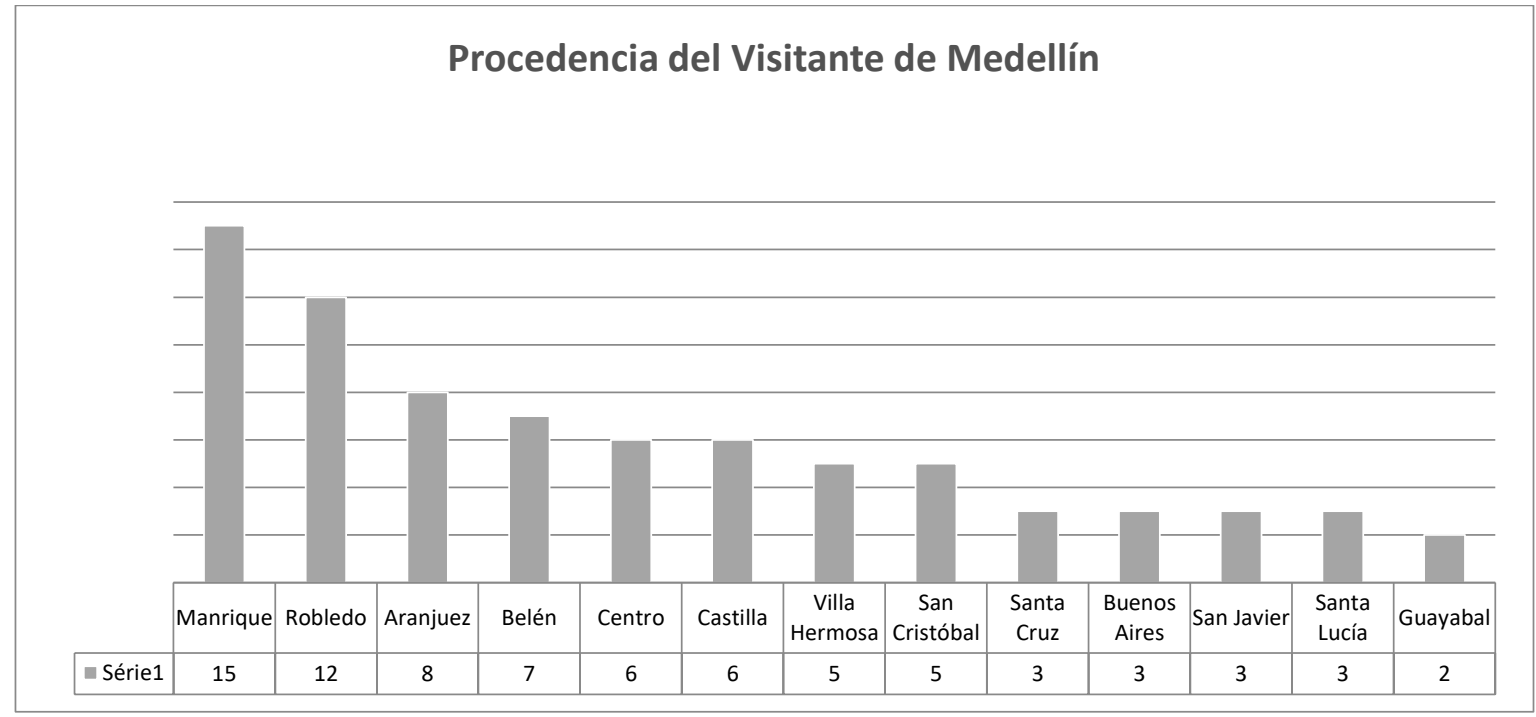

\subsection{PERFIL PÚBLICO NACIONAL}

Perfil 1: Hombres y mujeres de la Ciudad Capital del País- Bogotá. Asisten al Museo por curiosidad, porque es famoso, por conocer las obras expuestas. Generalmente están en la región por vacaciones o por trabajo. Tienen entre 18 y 34 años.

\section{- Lugar de procedencia del visitante Nacional}

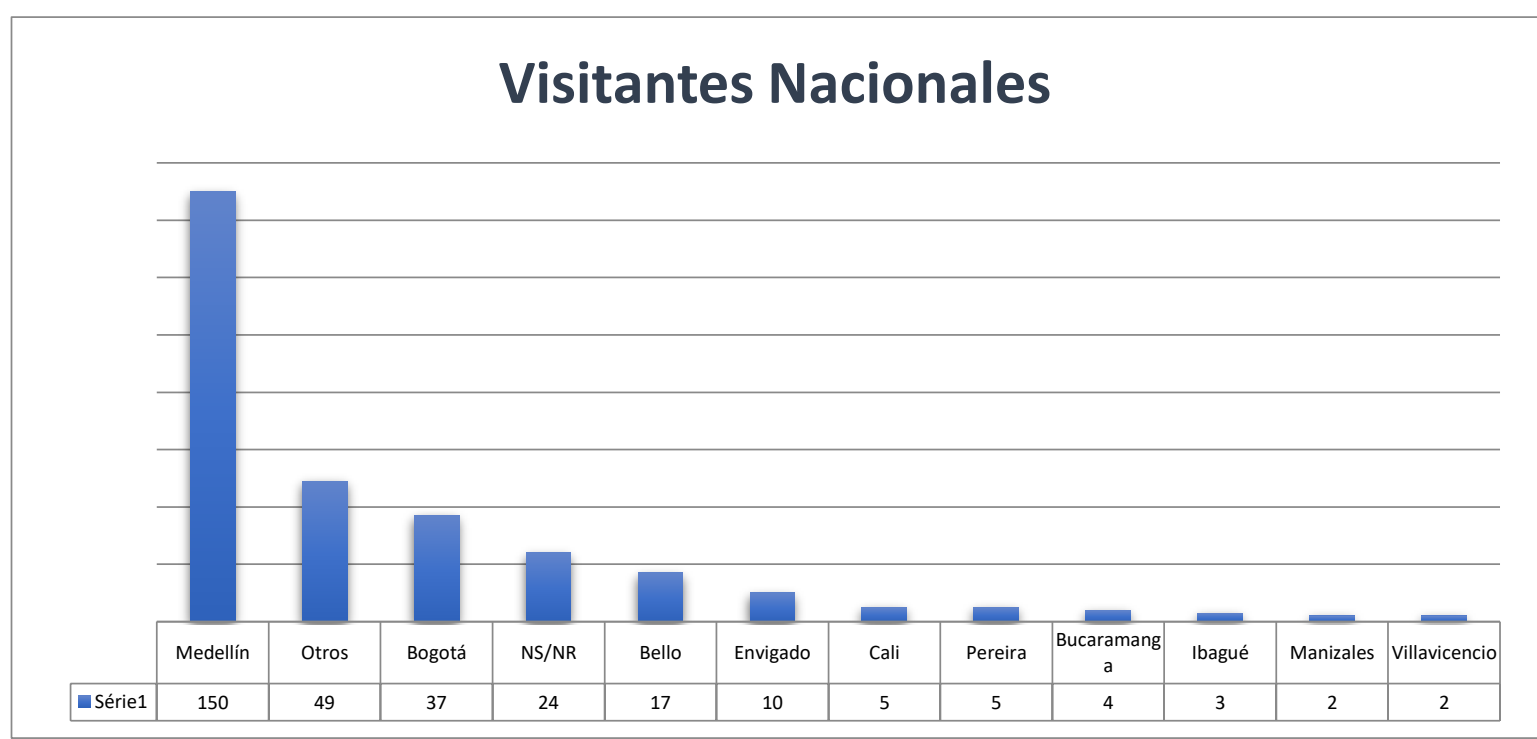


El 49\% de los que visitan el Museo son de Medellín, seguidos de los procedentes de Bogotá(12\%). Los visitantes del Municipio de Bello, también presentan cierta frecuencia de visita (6\%), es de señalar que estos datos coinciden con los reportados en el informe $2013^{4}$, en donde se encontró que el Museo es visitado principalmente por Colombianos (89\%) y que de estos el (50\%) eran de Medellín y el (14,25\%) de Bogotá. El dato sugiere estrategias para atraer visitantes de otras zonas del país y especialmente de otros Municipios de Antioquia, ya que como se indicó en el informe $2013^{5}$ hay muy baja presencia de visitantes del área Metropolitana y de otras regiones del Departamento.

\subsection{PÚBLICO EXTRANJERO}

Perfil 1. Mujeres y hombres entre 18 y 34 años, con Grado Universitario, de Maestría o PhD, en su mayoría empleados. Estos visitantes en su mayoría son solteros, proceden de México, EE.UU y Canadá. La ocupación de la mayoría es empelados o estudiante universitario.

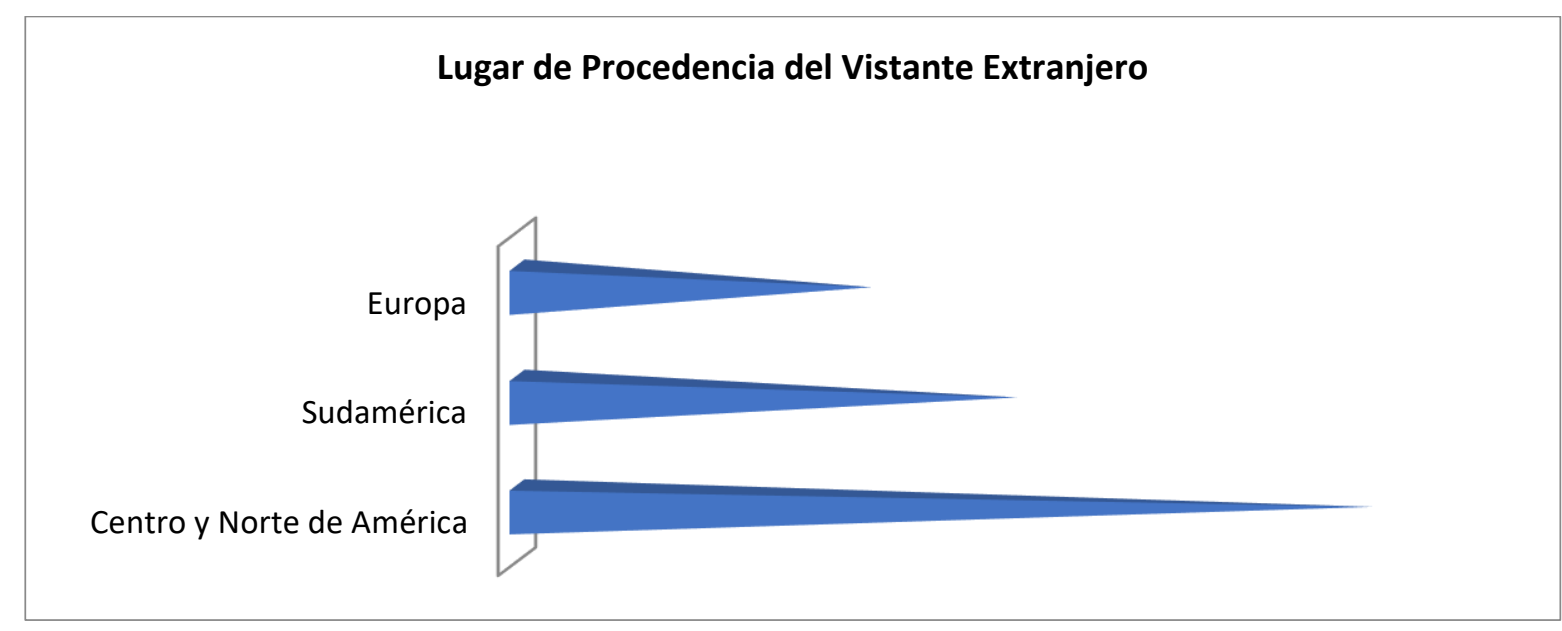

En comparación con el informe de $2013^{6}$, se encuentra una mejoría en cuanto a este ítem en tanto se amplió la visita de centro y norteamericanos, así como de suramericanos y europeos, es de señalar que los estadounidenses y los mexicanos son los visitantes más asiduos del Museo.

- Público extranjero potencial. Suramericanos, extranjeros jubilados: Los mayores de 55 son quienes menos visitan el Museo, una hipótesis de esto podría ser que no es el tipo de extranjero

\footnotetext{
${ }^{4}$ Informe "Análisis de Público Universitario para el Museo de Antioquia” (2013). Dra. Mónica Valle Flórez- Politécnico Colombiano Jaime Isaza Cadavid. Dra. Claudia Giraldo Velásquez. Dra. Sandra Zapata Aguirre. Institución Universitaria Colegio Mayor de Antioquia.

5 Idem

${ }^{6}$ En el Informe "Análisis de Público Universitario para el Museo de Antioquia” (2013), hizo encontró que la procedencia del público visitante del Museo, eran Colombianos (89\%) y que tan solo un (2\%) de Estados Unidos, y que el (1.0) procedían de Venezuela, Ecuador, España. Mónica Valle Flórez- Politécnico Colombiano Jaime Isaza Cadavid. Claudia Giraldo Velásquez. Sandra Zapata Aguirre. Institución Universitaria Colegio Mayor de Antioquia.
} 
que más visita Colombia. Se sabe que en Europa los mayores de 50 años son quienes más visitan museos. Otro público potencial son los extranjeros que viven en Medellín-Antioquia-Colombia.

La mayoría de visitantes extranjeros que van al Museo lo hacen por recomendación de amigos, son muy pocos los que indican que van al museo por la información suministrada en los guías turísticos de la Ciudad, o por la guía /libro de turismo. Los encuestados manifiestan que se enteran de las actividades culturales de la Ciudad por medio del email, las redes sociales y página Web del museo (12\%). Este público prefiere actividades de ocio, en las que tenga la oportunidad de aprender. Los nacionales prefieren la interacción social.

En la construcción del perfil psicográfico de los visitantes de museos, Kotler y Kotler (2001) \& Slater (2006) mencionan que las principales motivaciones a la hora de visitar museos son el escapismo, el aprendizaje y la interacción social ${ }^{7}$. Para Yellis (1985) las jerarquía de las 5 necesidades de Maslow: psicológicas, seguridad, social, autoestima y auto-actualización, influyen en las decisiones relacionadas con el ocio. Para el público joven y con un alto nivel educativo, las visitas a las galerías de arte moderno son una experiencia de ocio, motivada por el deseo de relajación y de entretenimiento ${ }^{8}$. En general para los turistas la visita a los museos es una actividad beneficiosa para ellos y sus hijos (Richards, 1999, 2001, 2002).

Otro tipo de perfil de visitantes del museo son los "asiduos" que representan el (57\%) y visitantes "nuevos" que llegan a representar el (43\%).

\section{CONCLUSIONES}

El estudio y observación de públicos de los museos se constituye en una importante estrategia en el "desarrollo de la audiencia" expresión que alude a mejorar los servicios para los visitantes: múltiples experiencias, autodesarrollo, mejorar la comunicación con estos, el acceso, interacción y segmentación de públicos. Dinámica necesaria para que los museos logren competir en la industria del entretenimiento, el ocio y el tiempo libre, puedan llevar a cabo su misión, la cual se hace más compleja al encontrar pocos recursos económicos para la cultura, además de nuevos públicos con diversas expectativas y motivaciones en torno a estos recintos.

Y es que la motivación reverencial y educativa de la visita a los museos, con las que Falk y Dierking (1992) caracterizaron a los públicos del Reino Unido en los 70 y 80, parece ya cosa del pasado,

\footnotetext{
${ }^{7}$ Slater, A. (2006). Escaping to the gallery: understanding the motivations of visitors to galleries. International Journal of Nonprofit and Voluntary Sector Marketing.

${ }^{8}$ Proyecto de Investigación Turismo Cultural, segmentando a los turistas que van en busca de la realización de un turismo cultural. Estudio adelantado por Richards en Europa para la Asociación para el Turismo y Educación de Ocio (ATLAS).
} 
la recreación social, esto es la diversión y el entretenimiento en un ambiente seguro con familia y amigos para ser lo que prima al momento de elegir en que ocupar el tiempo libre. En el estudio realizado se encuentra que la curiosidad, también aparece como motivación que lleva al nuevo público al museo. Público al que le molesta el silencio, quiere hablar con su familia y amigos, prefiere la interacción social más que la contemplación, la diversión al conocimiento.

En este contexto el museo denominado clásico tiene las horas contadas. Cambia o lo cambian. ¿Hay que cambiar al museo o las expectativas del público?, cada museo se lo responderá; por lo pronto, en relación al estudio efectuado y del que se da cuenta en esta ponencia, se encuentra que otros públicos visitan hoy el museo, lo que se constituye en reto y en una gran oportunidad para la cultura. Estudiantes, empleados y en general el estrato 1,2 y 3 de Medellín asisten, más frecuentemente al museo, no tantas veces como las estadísticas quisieran pero si se acercan más a estos recintos que en épocas pasadas. Quizás esto se deba a una política de entrada gratuita con recibo de servicios públicos.

Resulta interesante para este estudio encontrar que las empleadas domésticas así como los jubilados se tornan público emergente del museo estudiado. Hay que fidelizarles y atraer más público de estos. Entre los retos atraer nuevamente al museo al visitante experto, y al de estrato 4, 5 y 6 . Y es que el llamado público expertos, aquel que disfrutara el personal del museo, por su interés en una experiencia museística excepcional y de alto contenido cultural, es el que menos llega a este.

Una gran oportunidad evidenciar que aunque el público extranjero, que es el público ideal para este museo, en tanto es el que compra boleta de ingreso, su audiencia por desarrollar corresponde al público local y nacional. Una buena noticia que el museo no tienen género, tanto hombre como mujeres le visitan. Ello desde una clasificación clásica, ¿tendremos que preguntarnos por gay, lesbianas y grupos minoritarios que asisten al museo? Y por supuesto ¿si hay que ir al museo o sacar el museo?

Se encuentra en el estudio que los solteros van al museo más que los casados, también asisten más los universitarios de estratos 4,5 y 6; ¿Qué pasa con lo de estrato 1, 2 y 3 que asisten poco? La pregunta se lanzó en una clase con estudiantes universitarios de estos estratos, la respuesta, "yo prefiero irme al lleras [Zona rosa de Medellín] y tomarme una cerveza”. Sería recomendable generar estrategias para que este tipo de público se acerque al museo.

Fernández (2013) clasifica a los públicos de museos como: reales, potenciales y virtuales. Los potenciales serían los menos aptos para ir al museo: personas con limitaciones físicas, familias con niños pequeños, personas de bajos ingresos, minorías culturales o recién emigrados. Los virtuales los que interaccionan con los museos por medio de website. La definición de los visitantes de Museos como “extraños" es propia de los museos que se apegan a las funciones tradicionales de conservar, preservar y 
exhibir una colección, sin tener en cuenta a los visitantes. En este caso el museo se asocia a códigos que lo alejan de su publico: elevación moral, culturización, restricción horaria, etc. ${ }^{9}$

En el estudio realizado se identifican públicos potenciales en cada perfil tales como los extranjeros Suramericanos, los ejecutivos y profesionales locales y los profesores de arte. Son potenciales también las figuras del benefactor y el que hacer servicio social en el museo. Lo importante es determinar ¿qué públicos? ¿ por qué? y ¿para qué?. Se requiere estrategia para desarrollar el público virtual.

Se concluye que el museo estudiado, cumple una función social y cultural importante en la región antioqueña, que sus estrategias educativas, comunicativas y de gestión ha permitido ampliar el acceso a grupos sociales poco favorecidos como son los estratos 1,2 y 3 . Estas estrategias financiadas directamente por los entes gubernamentales de la región, no son suficientes para el sostenimiento de este recinto, por lo debe desarrollar públicos, servicios, acciones en sí estrategias que le permitan ampliar el perfil de sus públicos y sus ingresos.

${ }^{9}$ ECD. Ministerio de Educación, Cultura y Deporte (2010). Conociendo a todos los públicos. ¿Qué imágenes se asocian a los museos? España. http://www.gestorcultural.org/images/noticies/noticia227172512.pdf 


\section{BIBLIOGRAFÍA}

ASENSIO \& Pol (2007). Conversaciones sobre el aprendizaje informal en museos y el patrimonio. En Turismo, Patrimonio y Educación, Fernández Betancort, editor. Escuela Universitaria de Turismo de Lanzarote Adscrita a la Universidad de Las Palmas de Gran Canaria. Este libro se puede descargar en español y en ingles en la siguiente dirección como un E-BOOK. Colección Pasos Edita "REVISTA DE TURISMO Y PATRIMONIO CULTURAL" http://www.pasosonline.org/

CAPRIOTTI, P. (2013). Maniging Strategic Communication in Museums. The case of Catalan museums. Communication \& Society/ Comunicación y Sociedad, Vol. 26, n. 3, pp. 98-116).

ECD. Ministerio de Educación, Cultura y Deporte (2010). Conociendo a todos los públicos. ¿Qué imágenes se asocian a los museos? http://www.gestorcultural.org/images/noticies/noticia227172512.pdf

HOOD, M. (1981). Staying away, Why people choose not to visit museums? Museum News.

RODRÍGUEZ, Ángel (2004), “Comunicación para estimular la visita al museo”, en: Museo, núm. 9, pp. 115- 124. En Alarcón, R. (2007). Sociología y estudios de público en los museos españoles. Revista Museo. X Jornadas de Museología- España. Consutlado 13/08/2015. Disponible en http://www.apme.es/revista/museo12_233.pdf

(2002), "Nueva sociedad, nuevos museos. El papel del marketing en los museos", en: Revista de Museología, núms. 24-25, pp. 25-38.

SLATER, A. (2006). Escaping to the gallery: understanding the motivations of visitors to galleries. International Journal of Nonprofit and Voluntary Sector Marketing. Published online 13 December 2006 in Wiley InterScience.

PINCUS J. 2004. The consequences of unmet needs: the evolving role of motivation in consumer research. Journal of Consumer Behaviour 3(4):375-387.

VALLE, M. \& Otros (2005). Comunicación Organizacional. Abordajes y Perspectivas de análisis. Centro Internacional de Estudios Superiores de Comunicación para América Latina, CIESPAL.

VALLE, M. (2014). La investigación en comunicación en Antioquia Colombia. Revista Luciérnaga/ Comunicación, Año 6, N12. Facultad de Comunicación Audiovisual- Politécnico Colombiano Jaime Isaza Cadavid-PCJIC \& Facultad de Ciencias de la Comunicación - Universidad Autónoma de San Luis PotosíUASLP. México. ISSN 2027- 1557. Págs. 73-91.

VALlE, M; GIRALDO, C; ZAPATA, S. (2018). Museo de Antioquia. Públicos, estrategias y retos. Editorial Corporación Universitaria Remington- Medellín - Colombia. Disponible en: https://www.politecnicojic.edu.co/images/downloads/publicaciones/museo-antioquia/museo-deantioquia-publicos-estrategias-y-retos.pdf

WALTL, Christian (2006). Museums for visitors: Audience development - A crucial role for successful museum management strategies. INTERCOM. Conference Paper. Consultado el 11/08/2015. Disponible en http://www.intercom.museum/documents/1-4waltl.pdf 